\title{
The value of $\mathrm{N}$-terminal pro-B-type natriuretic peptide (NT Pro-BNP) to predict sudden cardiac death in chronic heart failure patients with normal renal function; a systematic review and meta-analysis
}

\author{
Mehrdad Sheikhvatan $^{1}$, Zhaleh Ataei $^{2^{*} \mathbb{D}}$, Aigin Heydari $^{2}$, Aryan Zahergivar $^{2}$ \\ ${ }^{1}$ Neurology Department, Heidelberg University, Heidelberg, Germany \\ ${ }^{2}$ Department of Medicine, Shiraz University of Medical Sciences, Shiraz, Iran
}

\section{A R T I C L E I N F 0}

Article Type:

Meta-analysis

Article History:

Received: 10 May 2018

Accepted: 18 June 2018

ePublished: 27 July 2018

\section{Keywords:}

B-type natriuretic peptide

Sudden cardiac death

Chronic heart failure

Meta-analysis

\begin{abstract}
A B S T R A C T
Context: The role of N-terminal pro-B-type natriuretic peptide (NT pro-BNP) has been understood as an important and critical biomarker in the diagnosis and predicting poor outcome of cardiac dysfunction.

Objectives: We aimed to systematically review the papers on the value of NT pro-BNP in prediction of sudden cardiac death (SCD) in chronic heart failure (CHF) patients.

Data Sources: This study was based on the Systematic Reviews and Meta-analysis (PRISMA) study reporting system.

Study Selection: During the deep searches using the keywords, 67 studies were initially considered for primary assessment. Of those, 12 were completely matched with the study endpoint. In final, six were excluded because of unavailability of full texts or acquired data and thus six studies were finally analyzed.

Data Extraction: Two researchers independently used the key words "chronic heart failure, B-type natriuretic peptide, sudden cardiac death" and their combination and searched the national and international databases including Scopus, PubMed, Science Direct, Web of Science, Springer, and the Google Scholar search engine.

Results: The pooled prevalence of SCD in CHF patients was found to be $6.9 \%$ (95\% CI: $5.2 \%$ to $9.0 \%)$. The statistical heterogeneity was high with an $\mathrm{I}^{2}$ of 84.488 . Abnormal elevated level of NT pro-BNP was significantly associated with the increased risk for SCD with a hazard ratio of 4.2 (95\% CI: 2.2 to 8.7 ).

Conclusion: Measuring the serum level of NT pro-BNP in CHF patients can be valuable to predict long-term SCD. In this regard, significant elevation of this biomarker may be associated with the four-fold risk of SCD in such patients.
\end{abstract}

Implication for health policy/practice/research/medical education:

Measuring the serum level of N-terminal pro-B-type natriuretic peptide (NT Pro-BNP) in chronic heart failure (CHF) patients can be valuable to predict long-term sudden cardiac death (SCD). In this regard, significant elevation of this biomarker may be associated with the four-fold risk of SCD in such patients. A few documents are available in the best cutoff value of this marker to achieve this goal with high sensitivity and specificity and thus more cross-sectional and cohort studies should be performed with the aim of yielding the best cutoff points of the marker.

Please cite this paper as: Sheikhvatan M, Ataei Z, Heydari A, Zahergivar A. The value of N-terminal pro-B-type natriuretic peptide (NT Pro-BNP) to predict sudden cardiac death in chronic heart failure patients with normal renal function; a systematic review and meta-analysis. J Nephropharmacol. 2018;7(2):164-168.

\section{Introduction}

Chronic heart failure (CHF) is a multiplex syndrome characterized by both functional and structural abnormalities in myocardial tissue leading ventricular functional impairment and thus failure in cardiac blood supplying process $(1,2)$. Not only myocardial dysfunction can result in $\mathrm{CHF}$, but any defect in other parts of heart such as cardiac valvular system, pericardium, and even great vessels along or in combination with together can lead the cardiovascular system to failure (3). The 
pathogenic mechanisms of CHF are very complex. However a combination of mechanical, hormonal, neuronal, cellular and even genetic factors contributes to the pathogenic fundament of $\mathrm{CHF}(4,5)$. In this regard, ventricular dysfunction-related ischemia, mechanical hemodynamic instability, accelerated cellular apoptosis, genetic predisposition, and hormonal stimulations have been identified as the main basis for CHF flaring (68 ). The central role of some biochemical and hormonal components in stimulating CHF or its deterioration has been recently highlighted.

About 25 years ago, the role of $\mathrm{N}$-terminal pro-B-type natriuretic peptide (NT pro-BNP) has been understood as an important and critical biomarker in the diagnosis and predicting poor outcome of cardiac dysfunction $(9,10)$. Since then, many studies have focused on the role of this marker to early predict mortality and morbidity in patients with progressive heart disease such as CHF. It seems that NT pro-BNP is high sensitive for predicting CHF exacerbation and even its cutoff values with maximized diagnostic value in such patients have been introduced (11). However, this diagnostic value can be influenced by some important underlying factors such as simultaneous renal insufficiency (commonly revealed in CHF patients) $(12,13)$, obesity (14-16), and underlying atrial fibrillation $(17,18)$ and thus this biomarker may not be adequately valuable in some patients' subgroup. Thus, providing a systematic conclusion of various studies on the value of NT pro-BNP in predicting CHF prognosis is necessary. Herein, we aimed to systematically review the papers on the value of NT pro-BNP in prediction of sudden cardiac death (SCD) in CHF patients.

\section{Materials and Methods \\ Data sources}

This study was based on the Systematic Reviews and Meta-Analysis (PRISMA) study reporting system (19). All articles about the value of NT pro-BNP in predicting outcome of CHF have been studied. Two researchers independently used the key words "chronic heart failure, B-type natriuretic peptide, sudden cardiac death" and their combination and searched the national and international databases including Scopus, PubMed, Science Direct, Web of Science, Springer, and the Google Scholar search engine. For generalization of the search step, internal and external keywords were used for general keywords and in external databases, the combination of keywords was used with the "AND" and "OR" operators. The search step was unrestricted and updated by 2018 .

\section{Study selection}

In order to complete the search process, a manual search was also performed by reviewing the sources of the articles found. The selection criteria included articles that reported the association between NT pro-BNP and CHFrelated sudden death. Exclusion criteria included studies that did not have sufficient data and information, studies which full text was not available, or studies that did not have the required quality.

\section{Data extraction}

Researchers examined the quality of papers using the STROBE checklist (20), a well-known international standard checklist for qualitative evaluation of articles. This checklist consists of 22 different sections and covers different parts of a report including sampling, variable measurement, statistical analysis, confounding modifications, validity and reliability of used tools and study objectives. The score range for this checklist is between 0 and 44, and articles that score less than 15.5 have been excluded from the study.

Studies that deserve the required quality were finally analyzed. To reduce reporting bias and error in data collection, two researchers independently extracted data from articles. The researchers listed a checklist including the name of the author, study title, the year and place of the study, the total number of patients, the study type, the details of the CHF outcome, and the value of NT pro-BNP indicated as odds ratio or by assessing the area under the ROC curve.

\section{Statistical analysis}

The variance of each study was calculated according to the binomial distribution. Studies were combined according to sample number and variance. Regarding the heterogeneity of the studies, the random effects model was used and in order to evaluate the studies, Cochran method and $\mathrm{I}^{2}$ index were used. The $\mathrm{I}^{2}$ less than $25 \%$ indicated a low heterogeneity, between $25 \%$ and $75 \%$ indicated a moderate heterogeneity and higher than $75 \%$ indicated a high heterogeneity. Data were analyzed using STATA version 11. The significance level of the tests was considered to be $P<0.05$.

Results

During the deep searches using the keywords, 67 studies were initially were considered for primary assessment. Of those, 12 were completely matched with the study endpoint. In final, six were excluded because of unavailability of full texts or acquired data and thus six studies were finally analyzed (21-26) (Table 1), which were published in the period from 2002 to 2013 . The total number of participants in the study was 7888 (4055 men and 3833 women, average age of 64.17 years, ranged 54 to 73 years). The mean follow-up time of the patients was overall 3.8 years ranged 1 to 12.5 years. The meta-analysis of the prevalence of SCD in CHF patients resulted from the combination of the results of studies presented in Figure 1. Based on the meta-analysis processing and assessing size effect and random effect models, the pooled prevalence of SCD in CHF patients was found to be $6.9 \%$ (95\% CI: $5.2 \%$ to $9.0 \%$ ). The statistical heterogeneity was 
Table 1. The details of the studies which systematically reviewed in the present study

\begin{tabular}{|c|c|c|c|c|c|c|c|c|c|c|}
\hline Author, year & No. of patients & Mean age & $M / F$ & NYHA & Follow-up & SCD & OR & Significance & Cut off & Sen/Spe \\
\hline Vrtovec, 2013 (21) & 398 & $65 \pm 12$ & $262 / 136$ & III-IV & $1 y$ & 20 & $0.7(0.3-1.9)$ & Negative & --- & --- \\
\hline Patton, 2011 (22) & 5447 & $73 \pm 6$ & $2255 / 3192$ & II-IV & $12.5 \mathrm{y}$ & 289 & $2.5(1.6-3.8)$ & Positive & --- & --- \\
\hline Bayes, 2007 (23) & 494 & $63 \pm 11$ & $385 / 109$ & II-IV & $3 y$ & 50 & $4.6(2.2-9.5)$ & Positive & 908 & $81 / 55$ \\
\hline Watanabe, 2006 (24) & 680 & $66 \pm 14$ & $469 / 211$ & I-IV & $2 y$ & 36 & $8.9(4.6-17.5)$ & Positive & 200 & $61 / 62$ \\
\hline Watanabe, 2005 (25) & 417 & $64 \pm 14$ & $289 / 1128$ & II-IV & $2.2 \mathrm{y}$ & 30 & $3.4(1.4-7.9)$ & Positive & --- & --- \\
\hline Berger, 2002 (26) & 452 & $54 \pm 10$ & $395 / 57$ & I-IV & $2 y$ & 44 & $4.8(2.9-11.8)$ & Positive & -- & --- \\
\hline
\end{tabular}

Abbreviations: SCD, Sudden cardiac death; OR, odds ratio; Sen/Spe, sensitivity and specificity.

high with an $\mathrm{I}^{2}$ of $84.488(P<0.001)$. There was also a significant publication bias as evidenced by either funnel plot asymmetry or Egger test $(P=0.001$ for all) (Figure 2). Of six studies which were finally assessed, five revealed a significant association between the elevated serum level of NT pro-BNP and increased likelihood of SCD in CHF patients (22-26). In this regard, abnormal elevated level of NT pro-BNP (more than 10\% of normal range) was significantly associated with the increased risk for SCD with a hazard ratio of 4.2 (95\% CI: 2.2 to 8.7$)$. Of six studies in meta-analysis processing, only two assessed the value of increased level of NT pro-BNP for SCD from survival status by the ROC curve analysis that led to different cutoff values of this biomarker as $>908 \mathrm{pg} / \mathrm{mL}$ and $>200 \mathrm{pg} / \mathrm{mL}$ as indicators for risk of SCD. As shown in Table 1, the first cutoff point yielded high sensitivity but low specificity, while the second cutoff point achieved partially low sensitivity and specificity.

\section{Discussion}

In the current systematic review, concluding the findings of the studies on the value of elevated level of NT pro$\mathrm{BNP}$ as a novel diagnostic biomarker for predicting risk for SCD in heart failure patients reached to demonstrate its high predicting value. In this regard, elevated level of NT pro-BNP at least $10 \%$ of normal range was link to more four-time risk for SCD in CHF patients and thus measuring serum level of this markers can successfully applied to estimate the risk for cardiac sudden death and also to stratify the patients to low, intermediate or high risk for this event. However, three important points

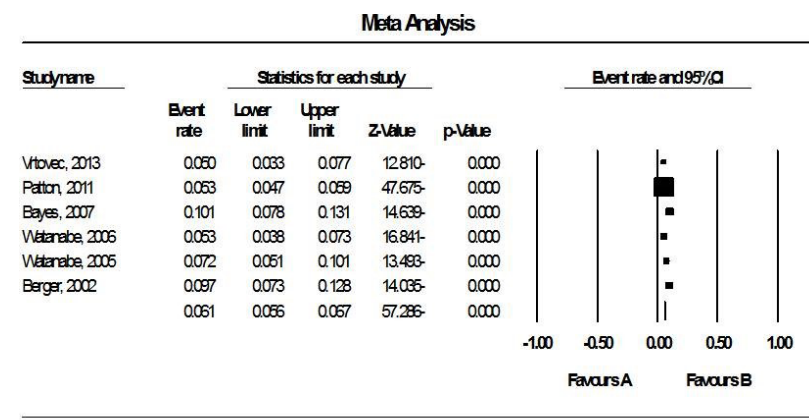

Figure 1. FOREST plot of the primary endpoint, overall sudden cardiac death related to $\mathrm{CHF}$. should be taken into consideration. First, a few studies have focused to estimate its value to discriminate survived from non-survived conditions (using the ROC curve analysis) and thus the best cutoff value for this marker to predict cardiac sudden death risk remains uncertain. In this regard, two studies with this parameter could not lead us to an appropriate cutoff point with the aim of yielding acceptable sensitivity and specificity. Second, we concluded high heterogeneity among the studies on our endpoint probably because of the considerable differences in study powers, type of the studies, inclusion or exclusion criteria, ignoring medications or other therapeutic interventions, and also the difference in the patients' functional classes. Summing these findings emphasizes us to the importance of further studies on the value of NT pro-BNP to predict CHF outcome and prognosis. Overall, the present evidence protects high power of this marker to predict mid-term or long-term SCD following $\mathrm{CHF}$ exacerbation. However, it seems that the combination of NT pro-BNP with other specific markers can increase our confidence to predict poorer outcome in CHF patients. As shown by Vrtovec et al (21), considering prolonged QT interval along with elevated level of NT pro-BNP can effectively estimate the likelihood of SCD in heart failure patients. Thus, it seems that the use of NT proBNP simultaneous with other markers can result in high specificity and confidence for predicting CHF improper outcome.

\section{Conclusion}

As the final conclusion, measuring the serum level of

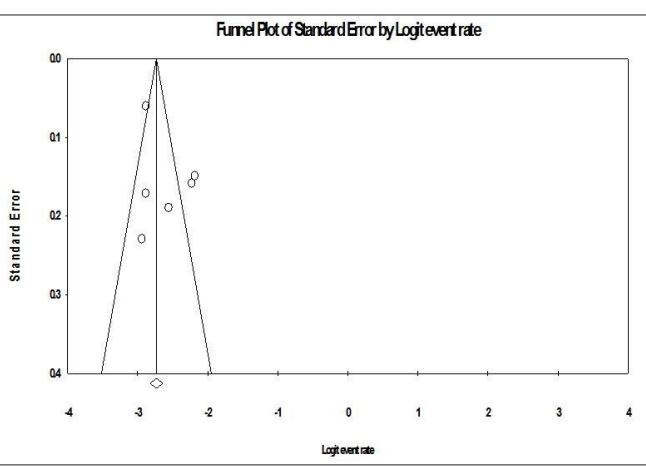

Figure 2. Funnel plot of the results on the publication bias. 
NT pro-BNP in CHF patients can be valuable to predict long-term SCD. In this regard, significant elevation of this biomarker may be associated with the four-fold risk of SCD in such patients. A few documents are available in the best cutoff value of this marker to achieve this goal with high sensitivity and specificity and thus more crosssectional and cohort studies should be performed with the aim of yielding the best cutoff points of the marker.

\section{Study limitations}

During the research, we encountered some problems such as inconsistencies in implementation and time constraints.

\section{Authors' contribution}

SHM and AZH designed the study, observed accuracy and validity of the study. ZA collected the data and follow the study. SHM, AZH and HA supervised the project. SHM, AZH, AND HA wrote the paper. All authors edited and revised the final manuscript and accepted its publication.

\section{Conflicts of interest}

The authors declared no competing interests.

\section{Ethical considerations}

Ethical issues (including plagiarism, data fabrication, double publication) have been completely observed by the authors.

\section{Funding/Support}

The authors declare that there is no source of funding for the research reported.

\section{References}

1. Dassanayaka S, Jones SP. Recent developments in heart failure. Circ Res. 2015;117:e58-63.

2. Inamdar AA, Inamdar AC. Heart failure: diagnosis, management and utilization. J Clin Med. 2016;5:72-6.

3. Hopper I, Easton K. Chronic heart failure. Aust Prescr. 2017;40:128-36. doi: 10.18773/austprescr.2017.044

4. Saccà L. Heart failure as a multiple hormonal deficiency syndrome. Circ Heart Fail. 2009;2:151-6

5. Lopes LR, Elliott PM. Genetics of heart failure. Biochim Biophys Acta. 2013;1832:2451-61.

6. Seixas-Cambão M, Leite-Moreira AF. Pathophysiology of chronic heart failure. Rev Port Cardiol. 2009;28:439-71.

7. Palazzuoli A, Nuti R. Heart failure: pathophysiology and clinical picture. Contrib Nephrol. 2010;164:1-10. doi: $10.1159 / 000313714$

8. Metra M, Teerlink JR. Heart failure. Lancet. 2017; 390:1981-1995. doi: 10.1016/S0140-6736(17)31071-1.

9. Richards AM. N-Terminal B-type Natriuretic Peptide in Heart Failure. Heart Fail Clin. 2018;14:27-39. doi: 10.1016/j.hfc.2017.08.004.

10. Malhotra AK, Ramakrishna H. N-terminal pro B type natriuretic peptide in high cardiovascular-risk patients for noncardiac surgery: What is the current prognostic evidence? Ann Card Anaesth. 2016;19:314-20. doi:
10.4103/0971-9784.179636.

11. Alkhawam H, El-Hunjul M, Nguyen J, Desai R, Syed U, Vittorio TJ. Natriuretic peptide hormones in congestive heart failure: challenges, clinical interpretation and review of studies. Acta Cardiol. 2016;71:417-24. doi: 10.2143/ AC.71.4.3159694.

12. McCullough PA, Duc P, Omland T. B-type natriuretic peptide and renal function in the diagnosis of heart failure: an analysis from the Breathing Not Properly Multinational Study. Am J Kidney Dis. 2003;41:571-9.

13. Chenevier-Gobeaux C, Claessens YE, Voyer S, Desmoulins $D$, Ekindjian OG. Influence of renal function on $\mathrm{N}$-terminal pro-brain natriuretic peptide (NT-proBNP) in patients admitted for dyspnoea in the Emergency Department: comparison with brain natriuretic peptide (BNP). Clin Chim Acta. 2005; 361:167-75. doi: 10.1016/j. cccn.2005.05.021

14. McCord J, Mundy BJ, Hudson MP. Relationship between obesity and B-type natriuretic peptide levels. Arch Intern Med. 2004;164:2247-52.

15. Wang TJ, Larson MG, Levy D, Benjamin EJ, Leip EP, Wilson PW, et al. Impact of obesity on plasma natriuretic peptide levels. Circulation. 2004;109:594-600. doi: 10.1161/01.CIR.0000112582.16683.EA.

16. Horwich TB, Hamilton MA, Fonarow GC. B-type natriuretic peptide levels in obese patients with advanced heart failure. J Am Coll Cardiol. 2006;47:85-90.

17. Knudsen CW, Omland T, Clopton P. Impact of atrial fibrillation on the diagnostic performance of B-type natriuretic peptide concentration in dyspneic patients: an analysis from the breathing not properly multinational study. J Am Coll Cardiol; 2005;46:838-44.

18. Ellinor PT, Low AF, Patton KK, Shea MA, Macrae CA. Discordant atrial natriuretic peptide and brain natriuretic peptide levels in lone atrial fibrillation. J Am Coll Cardiol. 2005;45:82-6.

19. Moher D, Shamseer L, Clarke M, Ghersi D, Liberati A. Preferred reporting items for systematic review and meta-analysis protocols (PRISMA-P) 2015 statement. Systematic Rev J 2015;4:1-9

20. Von Elm E, Altman D, Egger M, Pocock S, Gøtzsche P, Vandenbroucke J. Strengthening the Reporting of Observational Studies in Epidemiology (STROBE) statement: guidelines for reporting observational studies. BMJ. 2007;335:806-8. doi: 10.1136/bmj.39335.541782.AD.

21. Vrtovec B1, Knezevic I, Poglajen G, Sebestjen M, Okrajsek R, Haddad F. Relation of B-type natriuretic peptide level in heart failure to suddencardiac death in patients with and without QT interval prolongation. Am J Cardiol. 2013;111:886-90.

22. Patton KK, Sotoodehnia N, DeFilippi C, Siscovick DS, Gottdiener JS, Kronmal RA. N-terminal pro-B-type natriuretic peptide is associated with suddencardiac death risk: the Cardiovascular Health Study. Heart Rhythm. 2011;8:228-33. doi: 10.1016/j.hrthm.2010.10.038.

23. Bayes-Genis A, Vazquez R, Puig T, Fernandez-Palomeque C, Fabregat J, Bardají A, et al. Left atrial enlargement and NT-proBNP as predictors of sudden cardiac death in patients with heart failure. Eur J Heart Fail. 2007;9:802-7.

24. Watanabe J, Shiba N, Shinozaki T, Koseki Y, Karibe 
A, Komaru T, et al. Prognostic value of plasma brain natriuretic peptide combined with left ventricular dimensions in predicting sudden death of patients with chronic heart failure. J Card Fail. 2005;11:50-5.

25. Watanabe J1, Shinozaki T, Shiba N, Fukahori K, Koseki Y, Karibe A, et al. Accumulation of risk markers predicts the incidence of sudden death in patients with chronic heart failure. Eur J Heart Fail. 2006;8:237-42. doi: 10.1016/j. ejheart.2005.08.003.

26. Berger R, Huelsmann M, Strecker K, Moertl D, Moser P, Bojic A, et al. Neurohormonal risk stratification for sudden death and death owing to progressive heart failure in chronic heart failure. Eur J Clin Invest. 2005;35:24-31.

Copyright $\odot 2018$ The Author(s); Published by Society of Diabetic Nephropathy Prevention. This is an open-access article distributed under the terms of the Creative Commons Attribution License (http://creativecommons.org/licenses/by/4.0), which permits unrestricted use, distribution, and reproduction in any medium, provided the original work is properly cited. 